Fig. 3. Aus einem verticalen Schnitte durch die Kuppel der Follikel der Peyer'schen Plaques des Kaninchens. Gezeichnet wie früher. $F=$ Epithel mit einzelnen Lymphzellen und Haufen von denselben. Die Fettmoleküle imprägniren die einzelnen Lymphzellen und sammeln sich in Haufen. Dasselbe ist der.Fall mit der adenoiden Substanz $A$ der Follikel.

Die sämmtlichen Präparate sind nach Ueberosmiumsäure-Behandlung mit Picrocarmin gefärbt und in Canadabalsam eingeschlossen.

(Aus dem physiologischen Institut zu Christiania.)

\title{
Beiträge zur Kenntniss der Blutfarbstoffe.
}

\author{
Von
}

Assistent Jac. G. Otto.

Unter den Blutarten, aus welchen sich leicht und sicher grössere Quantitäten reines, krystallisirtes Oxyhämoglobin darstellen lässt, nimmt das Pferdeblut einen hervorragenden Platz ein, indem besagte Darstellung vielleicht hier noch besser gelingt als bei dem gewöhnlich benutzten Hundeblut. Während dieses aber sehr oft studirt ist, ist das Oxybämoglobin aus Pferdeblut, so viel mir bekannt, nur ein einziges Mal einigermassen genau untersucht und zwar von Hoppe-Seyler ${ }^{1}$ ). Die Ergebnisse dieser Untersuchung beziiglich der Elementarzusammensetzung des genannten Farbstoffes weichen ziemlich bedeutend von derselben der andern studirten Oxyhämoglobine $\mathrm{ab}$, indem der Stickstoffgehalt nach den von Kossel ausgeführten Analysen etwa 1\% höher als bei dem Hundehämoglobin ist. Es kann daher nur wünschenswerth sein, mehrere Analysen von diesem Körper anzustellen, um so mehr, als die von

1) Zeitschr. f. physiol. Chemie, II, p. 149-150. 
Kossel benuitzte Methode zur Bestimmung des Stickstoffgehalts (Dumas) noch nicht bei den übrigen Oxyhämoglobinen angewandt ist, und man deshalb nicht wissen kann, ob die gefundene Differenz von den verschiedenen Methoden und nicht von einer versehiedenen Zusammensetzung herrühre. Der Krystallwassergehalt, die Löslichkeitsverhältnisse und die optischen Eigenschaften des Pferdeoxyhämoglobin sind noch nicht Gegenstand einer Untersuchung gewesen, ebensowenig wie die darin enthaltene losgebundene Sauerstoffmenge entguiltig festgestellt ist. Ich habe deshalb den normalen Farbstoff des Pferdeblutes zum Gegenstand einer eingehenden Uutersuchung gemacht, dessen Resultat in dem folgenden Aufsatz mitgetheilt werden wird.

Die Darstellung des reinen Oxyhämoglobin geschah in der gewöhnlichen, für Hundeblut allgemein ïblichen Weise, und braucht daher nicht näher beschrieben zu werden; die erhaltenen Krystalle wurden erst nach viermaligem Umkrystallisiren für die Untersuchung verwendet.

Nach Funke krystallisirt das Pferdeoxylämoglobin im rhombischen System, während Hoppe-Seyler zwei Arten von Krystallen beschrcibt. Ich kann in dieser Beziehung die Resultate des letzterwähnten Forschers nur bestätigen, indem ich immer bei der ersten Krystallisation vorwiegend lange, dicke ${ }^{1}$ ) Prismen, sparsam mit kleinen Nadeln einer anderen Form gemischt, erhalten habe, Bei der Umkrystallisation dagegen gewinnen die letzten die Oberhand, so dass die Hauptmenge der Krystallmasse daraus besteht, und die genannten Prismen nur selten auftreten, es ist mir aber niemals gelungen, eine völlig gleichartige Krystallisation zu bekommen, weder von der einen, noch von der anderen Form.

Hoppe-Seyler giebt an, dass die erstgenannten Krystalle beim Auswaschen mit wasserhaltigem Alkohol verschwinden, welehes freilich theilweise der Fall ist, ich habe aber doch niemals in der Weise die Prismen völlig entfernen können.

Aus diesen Grüden hat Hoppe-Seyler die Bestimmung der Löslichkeitsverhältnisse und des Krystallwassergehaltes des Pferdeoxybämoglobin unterlassen, weil er annahm, dass die zwei Krystallformen sich in dieser Bezichung versehieden verhielten. Ich habe solche Bestimmungen obne irgend ein Resultat versucht,

1) Zuweilen sogar makroscopische. 
da die einzelnen gefundenen Werthe für den Krystallwassergehalt und die Löslichkeitsverhältnisse so stark differirten, dass ich mich völlig an Hoppe-Seyler's Anschaungen anschliesen und daraus folgern muss, was ich schon oben angedeutet habe, dass es mir niemals gelungen war, ein gleichartiges Product zu erhalten.

Die Elementaranalysen wurden mit Präparaten, die erst bei $0^{0}$ uiber Schwefelsäure im Vacuum und später bei $115^{\circ} \mathrm{C}$. im Wasserstoffstrom bis zum constanten Gewicht getrocknet waren, vorgenommen ${ }^{1}$ ). Der Controlle wegen habe ich ausser dem Stickstoffgehalt auch sämmtliche übrigen Elementarbestandtheile des Pferdeoxyhämoglobin bestimmt. Die einzelnen Bestimmungen geschahen in derselben Weise, wie früher bei der Elementaranalyse des Methämoglobin erwähnt. Nur bei den Stickstoffbestimmungen bin ich davon abgewichen, indem von den unten stehenden Analysen die 3 ersten nach Will-Varentrapp, die 2 letzten nach Dumas ausgefiuhrt wurden.

Die einzelnen analytischen Daten sind folgende:

1. Präparat.

\begin{tabular}{|c|c|}
\hline Angew. Substanz & 0,2480 \\
\hline Gef. Kohlensäure & 0,5432 \\
\hline$"$ Wasser & 0,1570 \\
\hline Angew. Substanz & 2,4876 \\
\hline Gef. Baryumsulfat & 0,1214 \\
\hline Angew. Substanz & 4,0866 \\
\hline Gef. Eisen & 0,0184 \\
\hline Angew. Substanz & 0,3022 \\
\hline Gef. Ammoniak & 0,0630 \\
\hline \multicolumn{2}{|c|}{ 2. Präparat. } \\
\hline Angew. Substanz & 0,2220 \\
\hline Gef. Ammoniak & 0,0461 \\
\hline
\end{tabular}

\section{Präparat.}

$\begin{array}{ll}\text { Angew. Substanz } & 0,3624 \mathrm{gr} \\ \text { Gef. Ammoniak } & 0,0757,\end{array}$

4. Präparat.

$\begin{array}{ll}\text { Angew. Substanz } & 0,2332 ” \\ \text { Gef. Stickstoff } & 0,0405 ”\end{array}$

5. Präparat.

$\begin{array}{ll}\text { Angew. Substanz } & 0,2888, \\ \text { Gef. Stickstoff } & 0,0506,\end{array}$

Aus den Analysen gehen folgende procentische Elementarwerthe hervor:

1) Wenn Hoppe-Seyler (1.c. p. 149) nur sagt, dass das von Dr. Kossel analysirte Pferdeoxyhämoglobin bei $0^{0}$ getrocknet war, ohne eine höhere Temperatur zu erwähnen, muss ich annehmen, dass dies nicht ganz korrect ist, da alle seine früheren Analysen von Blutfarbstoffen mit bei $110-115^{\circ}$ getrockneten Präparaten gemacht sind. 


\begin{tabular}{l|c|c|c|c|c|r} 
& I. & II. & III. & IV & V & Mittel. \\
Kohlenstoff & 54,76 & & & & & 54,76 \\
Wasserstoff & 7,03 & & & & & 7,03 \\
Stickstoff & 17,18 & 17,12 & $\mathbf{1 7 , 1 9}$ & 17,41 & 17,55 & 17,28 \\
Schwefel & 0,67 & & & & & 0,67 \\
Eisen & 0,45 & & & & & 0,45 \\
Sauerstoff & - & & & & & \\
& & & & & &
\end{tabular}

Um die Analysen von Kossel und von mir besser vergleichen zu können, sind sie unten zusammengestellt:

$\begin{array}{lrr} & \text { Otto. } & \text { Kossel. } \\ \text { Kohlenstoff } & 54,76 \% & 54,87 \% \\ \text { Wasserstoff } & 7,03 \% & 6,97 " \\ \text { Stickstoff } & 17,28 " & 17,31 " \\ \text { Schwefel } & 0,67 " & 0,65 " \\ \text { Eisen } & 0,45 " & 0,47 " \\ \text { Sauerstoff } & 19,81 " & -"\end{array}$

Die Uebereinstimmung hier ist so angenscheinlich, dass es nicht zu bezweifeln ist, dass Oxyhämoglobin aus Pferdeblut in der That mehr Stickstoff als die iubrigen untersuchten Hämoglobine enthält. Die Resultate meiner Stickstoffbestimmungen zeigen auch, dass die Methoden von Dumas und Will-Varrentrapp bei Hämoglobin so übereinstimmende Werthe geben, dass cine Wiederholung der Analysen der bis jetzt analysirten Blutfarbstoffe nicht nöthig ist. Nur für Hundeblutkrystalle sind bereits in dieser Beziehung Versuche gemacht, indem $\mathrm{F}$ ü fner ${ }^{1}$ ) den Stickstoffgehalt darin nach Dumas' Methode bestimmt und als Mittel aus 2 Analysen $16,47 \% \mathrm{~N}$ gefunden hat. Diese Zahl weicht, auch wenig von dem Durehsehnittwerth der zahlreichen Stickstoffsbestimmungen in Hundeoxyhämoglobin nach Will-Varentrapp $(16,25 \% \mathrm{~N})$ ab uud bestätigt somit meine Erfahrungen.

Es war nun nicht unwahrsehcinlich, dass der höhere Stickstoffgehalt des Pferdehämoglobin vielleicht auf die optischen Eigenschaften desselben in der Weise einwirken könnte, dass die spektrophotometrischen Constanten verschieden von den der übrigen Oxyhämoglobinen wären. Theils aus dem Grunde, theils weil eine

1) Zeitschr. f. physiol. Chemie, IV, p. 17, Anm. 
spektrophotometrische Untersuchung so vieler Blutfarbstoffe als möglich wïnschenswerth ist, habe ich eine solche unternommen, deren Resultat in der folgenden Tabelle mitgetheilt wird. Die Bestimmung der Constanten geschah in der iiblichen Weise und in den gewöhnlichen Spektralregionen (D32E-D54E und D63E-D84E), nur war der benutzte Apparat eine nette Modification des älteren Hüfner'schen Spektrophotometer, wesshalb die Werthe, wie ich in einem späteren Aufsatz zeigen werde, etwa höher ausgefallen sind.

\begin{tabular}{c||c||c}
\hline \hline $\mathrm{C}$ & $\mathrm{A}_{\mathbf{0}}$ & $\mathrm{A}_{0}^{\prime}$ \\
\hline 0,0028980 & 0,002025 & 0,001498 \\
0,0018812 & 0,001962 & 0,001442 \\
0,0016817 & 0,001908 & 0,001429 \\
0,0014910 & 0,001898 & 0,001401 \\
0,0012527 & 0,001859 & 0,001386 \\
0,0011604 & 0,001808 & 0,001345 \\
\hline Im Mittel. & 0,001910 & 0,001413
\end{tabular}

$$
\text { Quotient } \frac{A_{0}}{A_{0}^{\prime}}=1,352 \text {. }
$$

Für die Oxyhämoglobin aus Hundeblut ist bei demselben Apparat:

$$
\left.\begin{array}{l}
\mathrm{A}_{0}=0,001881 \\
\mathrm{~A}_{0}^{\prime}=0,001403
\end{array}\right\} \overline{\mathrm{A}_{0}^{\prime}}=134
$$

Die spektrophotometrischen Verhältnisse zeigen also eine so grosse Uebereinstimmung, dass man auch hier dieselbe färbende Gruppe wie in den übrigen untersuchten Hämoglobinen annehmen muss. Der Mehrgehalt an Stickstoff ist demnach wahrscheinlich dem im Hämoglobinmolekiil enthaltenen Globulincomponent zuzuschreiben.

Meinen Plan, die in Pferdeoxyhämoglobin losgebundene Sauerstoffmenge endgültig $\mathrm{zu}$ bestimmen, habe ich leider nicht realisiren können, weil ich gegenwärtig die dazu erforderlichen Apparate nicht besitze. In einer späteren Abhandlung beabsichtige ich deshalb diese Frage zum Gegenstande einer näheren Untersuchung zu machen. 